\title{
EDUCATION AND TRAINING Nutrition education in core medical curricula: a call to action from tomorrow's doctors
}

\author{
Authors: Jessica Ying-Yi Xie, ${ }^{A}$ Natalia Abramovich, ${ }^{B}$ Juliet Burridge, ${ }^{C}$ Ally Jaffee ${ }^{D}$ and Iain Broadley ${ }^{\mathrm{E}}$
}

In recent years lifestyle interventions have gained traction as increasingly useful ways to promote health among the population. Within these changes, nutrition remains paramount. We aimed to present an overview of the current status of nutrition education in UK undergraduate medical curricula, highlighting pockets of innovative teaching, alongside areas for improvement. Nutrition competency is outlined in General Medical Council Outcomes for Graduates, increasingly featured in mainstream headlines and relevant to each medical speciality. Drawing on our personal experiences we offer insights and suggestions on how the delivery and assessment of nutrition education could be improved. We believe that integrating nutrition into core curricula is urgently needed in order to increase students' and doctors' confidence in discussing the topic of nutrition with patients, with the aim to empower patients to make health promoting dietary changes.

KEYWORDS: nutrition, undergraduate medical education, nutrition in medical education, medical students, lifestyle medicine, improved health outcomes

DOI: $10.7861 /$ fhj.2020-0207

\section{Introduction - A hypothetical case study}

MT, a 55-year-old South Asian female patient, presents to general practice with a month-long history of tiredness. The GP conducts a thorough history, including a brief dietary history, revealing that MT often eats sugary and processed foods. The GP requests blood tests. At a follow-up appointment, the GP explains to MT that her HbA1c (glycated haemoglobin) is elevated, lying within the

Authors: Amedical student, University College London Medical School, London, UK; ${ }^{B}$ medical student, Barts and the London Medical

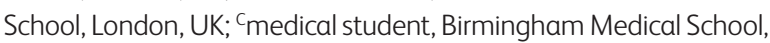
Birmingham, UK, and head of operations, Nutritank, Bristol Medical School, University of Bristol, Bristol, UK; Dmedical student, Bristol Medical School, Bristol, UK, and co-founder, Nutritank, Bristol Medical School, University of Bristol, Bristol, UK; Ejunior doctor, Worthing Hospital, Western Sussex Hospitals, NHS Foundation Trust, West Sussex, UK, and co-founder, Nutritank, Bristol pre-diabetes range, suggesting that this could be the reason why she has been feeling tired. MT enquires how she can reduce her future risk of developing type 2 diabetes mellitus (T2DM) and whether she needs to start taking medication. The GP suggests to first try dietary modification and increasing physical activity to reduce her blood sugar levels, and together they set such goals. MT is pleased that the GP is listening to her concerns and reassured that the lifestyle modifications advised are likely to make her feel less tired.

To support this, the GP refers MT to the practice's monthly community cooking workshop for patients who are at high risk of T2DM and cardiovascular disease. The workshop is run by a multiprofessional team consisting of GPs, dietitians, diabetes nurse specialists and a chef. The custom-made recipes are culturally appropriate for the local population, which has a high proportion of South Asian patients. Simple ingredient swaps such as replacing white rice with brown rice are advised. Local supermarkets are also supporting the programme, offering subsidised ingredients. A supportive local technology start-up company has produced an app which participants can use to share recipes, tips and support one another via the community forum. It is hoped that this programme will be rolled out into similar communities nationwide. Later that week, the GP delivers a tutorial on continuity of care across primary and secondary care for junior doctors in the deanery and presents MT as a case study, using this as an opportunity to educate the trainees about the inclusive and evidence-based community cooking programme. The GP reminds the junior doctors to include a suggestion for referral of patients at high risk of T2DM to the programme in their secondary care discharge notes to ensure community follow-up.

This hypothetical case study is one example of how healthcare professionals (HCPs) could apply principles of nutrition to routine clinical care, highlighting the benefit to patients like MT, with lifestyle-related chronic conditions. The status of nutrition education in medical curricula will be addressed below.

\section{Nutrition education in undergraduate medical curricula: Where are we now?}

The General Medical Council (GMC) publishes guidelines pertaining to the competencies expected from UK medical school graduates.'

However, there are no set quantities and qualities that medical school faculties are obliged to include for nutrition education in core curricula. We believe that there is a need for standardisation in curricula because the National Institute for Health and Care Excellence (NICE) recommends that lifestyle interventions, including 
dietary modification, are first-line in the prevention and management of common chronic medical conditions including T2DM. ${ }^{2}$

In response, we set up Nutritank, a think-tank led by medical students and junior doctors promoting the need for greater lifestyle medicine within healthcare training. A recent study ${ }^{3}$ aimed to gather the views of medical students and doctors on nutrition education and practice: over $95 \%$ of study participants believed that doctors play an important role in providing nutritional care, yet $70 \%$ of respondents reported receiving fewer than 2 hours of nutrition teaching while at medical school. Lack of knowledge was reported as the most common barrier to providing nutritional advice for patients. However, to the authors' knowledge, a comprehensive review of the state of nutrition education across the UK has not yet been performed. Thus, it is unknown where exactly the gaps in nutrition education lie.

In response to the increasing body of literature suggesting that nutrition education in UK undergraduate medical curricula is limited, ${ }^{3,4}$ we were inspired to organise a conference, inviting HCPs and scientists to deliver lectures and workshops on nutritional science and its application to clinical practice. More recently, we have been working with a multidisciplinary team (MDT) of primary and secondary care HCPs to deliver a national webinar series, 'Fil the gap', to teach the core principles of nutrition required to fulfil the competencies of qualified medical doctors as outlined in the GMC's Outcomes for Graduates.' The webinars are facilitated by medical students who lead a Nutritank branch at their respective medical school. There are currently 25 Nutritank branches across UK medical schools, and over 500 medical students and doctors signed up to the webinar series, suggesting that there is indeed interest.

However, we believe that, to be effective, nutrition teaching must be delivered primarily within core undergraduate medical curricula, as suboptimal diets are now known to be the leading cause of death globally, surpassing tobacco smoking. ${ }^{5}$ Understanding the principles of nutrition and diet is fundamental to clinical practice and reinforcing this early on in one's medical career may increase confidence in addressing patients' dietary needs.

There is limited understanding of the barriers to increasing the quantity and improving the quality of nutrition education in the UK. In one questionnaire study, ${ }^{4}$ academic representatives of UK medical schools reported a 'lack of prioritisation', and '[an inability] to devote more teaching time to nutrition' alongside 'difficulty organising topics and teaching sessions' as barriers towards improved nutrition education.

However, University College London Medical School (UCLMS) has integrated nutrition education into the core undergraduate curriculum without compromising aspects of the existing curriculum. ${ }^{6}$ UCLMS introduced a culinary medicine (CM) course in September 2019. CM is an innovative method of teaching HCPs about nutrition and its application to clinical practice through hands-on culinary skills training, case-based discussion and motivational interviewing role play. Students prepared meals according to the dietary requirements of specific patient case studies, for example, using ingredients with low glycaemic index for a patient with polycystic ovary syndrome. This exercise highlighted to us the potential barriers to meal preparation that patients who have dietary needs may face, and further consolidated the relationship between food and health and wellbeing.

Another strategy is to appoint a nutrition lead within the medical teaching faculty, who is responsible for organising the delivery, quality assurance and integration of nutrition education into the curriculum. Research demonstrates that medical schools who have appointed a nutrition lead have more time allocated to structured nutrition teaching. ${ }^{4}$ To date, two UK medical schools have appointed a dietitian as nutrition lead, including Brighton and Sussex Medical School (BSMS), ${ }^{7}$ where nutrition is taught within systems-based modules such as cardiology. Students are also offered research opportunities supervised by their nutrition lead. We believe that this is an excellent strategy to emphasise the importance of nutrition within the curriculum, and encourage medical schools to appoint the role of nutrition lead to a dietitian or faculty member with expertise in clinical nutrition and an understanding of how to optimise nutrition education.

A further example of successful integration of nutrition education into the undergraduate medical curriculum is Barts and The London medical school. ${ }^{8}$ Third year medical students take an online module in nutrition, a half-day of training in nutritional assessment and a half-day learning about barriers to healthy eating within the context of poverty. These sessions are delivered collaboratively by gastroenterologists, dietitians, primary care physicians and an organisation from the charity sector, providing medical students with an overview of nutrition in primary and secondary care settings.

\section{Why teach nutrition education to undergraduate medical students?}

Nutritional care is relevant to every medical specialty. While it is important to remember that dietary modification is not a replacement for, but rather complements, medical management, nutrition interventions are employed in primary care to prevent and manage common conditions, such as $\mathrm{T}_{2 \mathrm{DM}}{ }^{2}$ and in secondary care, nutrition can influence prognosis, such as determining post-operative outcomes. ${ }^{9}$

Moreover, the literature suggests that those who receive nutrition education become advocates for healthy eating, including adopting healthier eating habits themselves. ${ }^{10}$ We believe that our confidence in explaining what constitutes a balanced diet to patients has improved because we are able to draw inspiration from our own personal experiences. For example, we have expanded our knowledge through enrolling on extracurricular courses, attending conferences and engaging with HCPs on clinical placement who discuss nutrition with their patients. For those of us who have completed the CM module, we have learnt practical skills to prepare nutritious and cost-effective meals for ourselves, while simultaneously relating these skills to a clinical context which has the potential to achieve better patient and practitioner health.

\section{Nutrition education for tomorrow's doctors}

'Tell me and I forget, teach me and I may remember, involve me and I learn' (Benjamin Franklin)

Nutrition teaching can be delivered through a number of methods, including didactically. However, the literature, ${ }^{3}$ and our own learning experiences, suggest that engaging in active discussions, for example through consultation skills workshops and seminars, creates a more memorable learning experience.

Throughout our undergraduate medical training there has been little emphasis on nutritional interventions, which are often mentioned as an afterthought on the last slide of a lecture 
presentation. We believe that teaching on the management of conditions should mirror the NICE guidelines: nutritional interventions ought to be presented first, before medical or surgical interventions, to emphasise that lifestyle interventions are first-line.

\section{Integrating nutrition education into existing curricula}

We believe that an integrated approach to nutrition education, rather than a standalone module, would be more beneficial to allow students to appreciate the relevance of nutrition and its application to each clinical specialty. A spiral curriculum, which involves repeatedly revisiting topics throughout undergraduate medical training, would reinforce the importance of preventative nutrition in medicine and consolidate learning through repetition.

\section{Multidisciplinary teaching}

Furthermore, we feel that it is important for medical students to receive nutrition teaching from a multiprofessional team to understand the different roles and responsibilities of each HCP in ensuring appropriate nutrition for patients. Interprofessional education has positive outcomes for students: it provides a realistic model for future work in a healthcare setting ${ }^{11}$ and fosters an understanding of the value of each member of the team whilst challenging profession-specific stereotypes. ${ }^{12}$ Future research evaluating the effectiveness of interprofessional education specifically for nutrition education at medical school, could improve how we teach and assess nutritional knowledge.

\section{Nutrition education within a sociocultural framework}

As emphasised in the hypothetical case study in our introduction, we recognise that nutrition education should be embedded within sociocultural frameworks, as behavioural interventions that are not culturally informed may not succeed. ${ }^{13}$ An increased understanding of appropriate dietary recommendations for specific patient groups, alongside knowledge of the impact of the food environment on physical and mental health, mark important first steps towards providing person-centred care, which could improve long-term health outcomes.

\section{Assessment}

We believe that it would be most appropriate for students to be assessed practically through objective structured clinical examination (OSCE) assessments on taking a dietary history and communicating simple dietary advice to patients, as this simulates real-life situations in clinical practice. Malnutrition is estimated to be as high as $30 \%$ in UK hospitals. ${ }^{14}$ Doctors play a vital role in the recognition, prevention and treatment of malnutrition through conducting a nutritional assessment, which we feel is an essential clinical skill and a necessary component of a full clerking.

\section{Conclusion}

In summary, regarding nutrition education in UK undergraduate medical curricula, we believe that there is a need for improvement. While pockets of innovation and integrated teaching exist, such as CM and MDT teaching, the literature and our varied learning experiences suggest disparity between medical curricula. As medical students and a junior doctor, we have helped identify the need for curricula improvement and are driving for change. Future research is vital in order to discover specific gaps in nutrition education in UK undergraduate medical curricula, as well as identify barriers to increasing nutrition education faced by members of the medical school faculty. Building an awareness of the importance of nutrition and lifestyle medicine amongst HCPs will provide them with the tools to empower their patients, like MT in our case study, to live healthier lives.

\section{References}

1 General Medical Council. Outcomes for graduates. GMC, 2018. www.gmc-uk.org/-/media/documents/dc11326-outcomes-for-graduates-2018_pdf-75040796.pdf_[Accessed 24 September 2020].

2 National Institute for Health and Care Excellence. Type 2 diabetes in adults: management. (NICE guideline [NG28]). London: NICE, 2015. www.nice.org.uk/guidance/ng28 [Accessed 24 September 2020].

3 Macaninch E, Buckner L, Amin P et al. Time for nutrition in medical education. BMJ Nutr Prev Health 2020;3:40-8.

4 Long W, Neild P. PTH-133 Nutrition training in UK medical undergraduate programmes - has the situation improved? Gut 2016;65(Suppl 1):A285-6.

5 Afshin A, Sur PJ, Fay KA et al. Health effects of dietary risks in 195 countries, 1990-2017: a systematic analysis for the Global Burden of Disease Study 2017. Lancet 2019;393:1958-72.

6 Xie JY-Y, Poduval S, Vickerstaff V, Park S. Cross-sectional questionnaire study to gather the teaching preferences and expectations of UK undergraduate medical students for culinary medicine learning. BMJ Open 2020;10:e036410.

7 MacAninch E, Mellor D. Dietitians working in Medical Education. https://blogs.brighton.ac.uk/erimn/2020/06/08/dietitians-workingin-medical-education/ [Accessed 24 September 2020].

8 Blythe J. Medical school teaching in nutrition - the Barts and The London experience. 2020. www.nutritank.com/medicalschool-teaching-in-nutrition-the-barts-and-the-london-experience [Accessed 24 September 2020].

9 Fearon KC, Luff R. The nutritional management of surgical patients: enhanced recovery after surgery. Proc Nutr Soc 2003;62:807-11.

10 Pang B, Memel Z, Diamant C et al. Culinary medicine and community partnership: hands-on culinary skills training to empower medical students to provide patient-centered nutrition education. Med Educ Online 2019;24:1630238.

11 Ray S, Udumyan R, Rajput-Ray M et al. Evaluation of a novel nutrition education intervention for medical students from across England. BMJ Open 2012;2:e000417.

12 Guraya SY, Barr H. The effectiveness of interprofessional education in healthcare: A systematic review and meta-analysis. Kaohsiung J Med Sci 2018;34:160-5.

13 Greenhalgh T, Clinch M, Afsar $\mathrm{N}$ et al. Socio-cultural influences on the behaviour of South Asian women with diabetes in pregnancy: qualitative study using a multi-level theoretical approach. BMC Med 2015;13:120.

14 Elia M. The cost of malnutrition in England and potential cost savings from nutritional interventions. BAPEN, 2015. www.bapen.org. uk/pdfs/economic-report-short.pdf [Accessed 17 January 2021].

Address for correspondence: Juliet Burridge, Birmingham Medical School College of Medical and Dental Sciences, University of Birmingham, Edgbaston, Birmingham BA15 2TT, UK. Email: juliet@nutritank.com

Twitter: @nutritank 